\title{
ON $T$ DIRECTION OF ALGEBROID FUNCTION DEALING WITH MULTIPLE VALUES
}

\author{
ZHAOJUN WU ${ }^{\otimes}$ and DAOCHUN SUN
}

\author{
(Received 8 October 2007)
}

\begin{abstract}
Using Ahlfors' theory of covering surfaces, we prove the existence theorem for the $T$ direction for algebroid functions dealing with multiple values which extends the results proved by Guo, Zheng and $\mathrm{Ng}$ and answers a question by Wang, Giao and the present authors.
\end{abstract}

2000 Mathematics subject classification: primary 30D30; secondary 30D35.

Keywords and phrases: algebroid function, $T$ direction, multiple values.

\section{Introduction and main results}

Let $w(z)$ be a $v$-valued algebroid function in $|z|<+\infty$, defined by an irreducible equation (see $[2,6])$

$$
A_{v}(z) w^{v}+A_{v-1}(z) w^{\nu-1}+\cdots+A_{0}(z)=0
$$

where $A_{v}(z), A_{v-1}(z), \ldots, A_{0}(z)$ are entire functions with no common zero.

The value distribution of meromorphic functions due to Nevanlinna (see [11]) was extended to the corresponding theory of algebroid functions by Selberg, Ullrich, and Valiron in the 1930s. The study of the singular direction for algebroid functions was raised by Valiron in 1938. Valiron [7] conjectured that there exists a Borel direction for any $\nu$-valued algebroid function of finite and positive order growth. It was proved by Rauch that there exists a direction such that the corresponding Borel exceptional set is a set of linear measure zeros. In 1969, Toda [5] proved that there is a direction such that the corresponding Borel exceptional values are countable. Later, Lü and $\mathrm{Gu}$ [3] proved a more precise result than Valiron [6] and Toda [5] - that there is a Borel direction and its corresponding Borel exceptional values are $2 v$. We recall Lü and Gu's result for convenience.

The first author was supported in part by Xianning University grants KT0623, KZ0629 and by NSF grant 10471048. The second author was supported in part by NSF grant 10471048 .

(C) 2008 Australian Mathematical Society 0004-9727/08 \$A2.00+0.00 
THEOREM 1.1 [3]. Suppose that $w(z)$ is a v-valued algebroid function of order $\lambda$ which is a finite and positive number. Then there exists a ray $\arg z=\theta$ which is called a Borel direction of order $\lambda$ of $w(z)$, such that

$$
\limsup _{r \rightarrow \infty} \frac{\log n(r, \theta, \varepsilon, a)}{\log r}=\lambda
$$

holds for any $0<\varepsilon<\pi$ and all $a$ in $\mathbb{C}_{\infty}:=\mathbb{C} \cup\{\infty\}$ with at most $2 v$ exceptions.

Recalling the definition of the Borel direction for algebroid functions, this characterization is only effective for the finite and positive order functions. When the order $\lambda=0$ or $\infty$, it is not better to use the order to characterize the growth of $w$. In this case, Zheng [13] considered the $T$-direction which gives another singular direction for the meromorphic function. We recall his definition as follows.

DEFINITION 1.2. Let $f(z)$ be a meromorphic function in the complex plane. A radial $\arg z=\theta$ is called a $T$ direction of $f(z)$, provided that given any $b \in \mathbb{C}_{\infty}$, possibly with the exception of at most two values of $b$, for arbitrary small $\varepsilon>0$,

$$
\limsup _{r \rightarrow \infty} \frac{N(r, \theta, \varepsilon, b)}{T(r, f)}>0
$$

A radial $\arg z=\theta$ is called a precise $T$ direction of $f(z)$ if, in the above expression, $N(r, \theta, \varepsilon, b)$ is replaced by $\bar{N}(r, \theta, \varepsilon, b)$.

The existence theorem for the $T$ direction for a meromorphic function $f(z)$ satisfying $\lim \sup _{r \rightarrow \infty} T(r, f) /(\log r)^{2}=\infty$ was recently obtained by Guo et al. [1]. For a $v$-valued algebroid function, its $T$ direction is defined in the same way as Definition 1.2 with the corresponding characteristic and counting functions introduced by Selberg for algebroid functions, provided that the maximum number of exceptional values permitted here is $2 v$ instead of 2 . Most recently, $\mathrm{Wu}$ [9] proved the existence of the $T$ direction for algebroid functions and obtained the following theorem.

THEOREM 1.3. Suppose that $w(z)$ is a v-valued algebroid function defined by (1.1) and satisfying

$$
\limsup _{r \rightarrow \infty} T(r, w) /(\log r)^{2}=+\infty .
$$

TShen there exists a ray $\arg z=\theta$ which is called a $T$ direction of $w(z)$, such that

$$
\limsup _{r \rightarrow \infty} \frac{N(r, \theta, \varepsilon, a)}{T(r, w)}>0
$$

holds for any $0<\varepsilon<\pi$ and all $a$ in $\mathbb{C}_{\infty}$ with at most $2 v$ exceptions.

When multiple values are considered, Wang and Gao [8] prove the following theorem. 
THEOREM 1.4. Suppose that $w(z)$ is a v-valued algebroid function satisfying $\lim \sup _{r \rightarrow \infty} T(r, w) /(\log r)^{2}=+\infty$, but that it does not grow so rapidly that the lower order is finite. Then there exists a ray $\arg z=\theta$ which is called a $T$ direction dealing with multiple values of $w(z)$, such that

$$
\limsup _{r \rightarrow \infty} \frac{\bar{N}^{l)}(r, \theta, \varepsilon, a)}{T(r, w)}>0
$$

holds for any $0<\varepsilon<\pi$ and all $a$ in $\mathbb{C}_{\infty}:=\mathbb{C} \cup\{\infty\}$ with at most $2 v$ exceptions. Here $l \geq 2 v+1$.

In Wang and Gao [8, Remark 2], the authors ask whether Theorem 1.4 also holds when the lower order is infinite. In this paper, we shall address this problem and prove the following theorem.

THEOREM 1.5. Let $w(z)$ be a v-valued algebroid function defined on the whole complex plane and satisfying (1.2). Then there exists a ray $L: \arg z=\theta$ such that, for arbitrary small $\varepsilon>0$,

$$
\limsup _{r \rightarrow \infty} \frac{\bar{N}^{l)}(r, \theta, \varepsilon, a)}{T(r, w)}>0
$$

holds for any given $a \in \mathbb{C}_{\infty}$, provided the maximum number $p$ of exceptional values satisfying the following relation:

$$
p= \begin{cases}4 v & \text { if } l=1, \\ 3 v & \text { if } l=2, \\ {\left[2 \frac{l+1}{l} v\right]} & \text { if } 3 \leq l \leq 2 v, \\ 2 v & \text { if } l \geq 2 v+1 .\end{cases}
$$

Theorem 1.5 was posed by the present authors in [10]. There, they proved that Theorem 1.5 is valid for $3 \leq l$. At present, Zuxing [14] has independently proved that Theorem 1.5 is also valid for $l \geq 2 v+1$. The following example implies that no smaller number can replace the maximum number $q=4 v$ of exceptional values when $l=1$.

EXAMPLE 1.6. Let

$$
z(\zeta)=\int_{0}^{\zeta} \frac{d t}{\sqrt{\left(1-t^{2}\right)\left(1-\rho^{2} t^{2}\right)}} ;
$$

here $\rho \in \mathbb{R}$ and $0<\rho<1$. Then the inverse function of $z(\zeta)$ (denoted by $\zeta(z)$ ) is a meromorphic function of finite and positive order growth, and $1,-1,1 / \rho,-1 / \rho$ are 
also multiple values of $\zeta(z)$ (see [11, p. 151]). Let $w(z)$ be an algebroid function defined by the irreducible equation

$$
\zeta(z) w^{v}-1=0
$$

By Theorem 1.5, $w(z)$ has at least one $T$ direction dealing with multiple values with respect to $l=1$, which has $q=4 v$ dealing with multiple values $\sqrt[v]{1}, \sqrt[v]{-1}, \sqrt[v]{\rho}, \sqrt[v]{-\rho}$.

\section{Some notation and lemmas for algebroid function}

For the sake of convenience, we introduce some basic notation for algebroid functions (see [2]). Suppose that $w(z)$ is a $v$-valued algebroid function defined by expression (1.1) on $|z|<\infty$. The single-valued domain of definition of $w(z)$ is a $\nu$-sheeted covering of $z$-plane, a Riemann surface, denoted by $\widetilde{R}_{z}$. It is denoted by $\widetilde{z}$ since the point in $\mathbb{R}_{z}$ whose projection in the $z$-plane is $z$. The part of $\widetilde{R}_{z}$ which covers a disk $|z|<r$ is denoted by $|\widetilde{z}|<r$. For any $a \in \mathbb{C}_{\infty}$, put

$$
\begin{aligned}
& N(r, a)=\frac{1}{v} \int_{0}^{r} \frac{n(t, a)-n(0, a)}{t} d t+\frac{n(0, a)}{v} \log r, \\
& m(r, w)=\frac{1}{2 \pi v} \int_{|\widetilde{z}|=r} \log ^{+}\left|w\left(r e^{i \theta}\right)\right| d \theta, \quad z=r e^{i \theta},
\end{aligned}
$$

where $n(r, a)$ is the number of zeros, counted according to their multiplicities, of $w(z)-a$ in $|\widetilde{z}| \leq r$. Suppose that $a=\infty$. Let

$$
T(r, w)=m(r, w)+N(r, w) .
$$

The Ahlfors-Shimizu characteristic may be written as

$$
\frac{1}{v} \int_{0}^{r} \frac{S(t, w)}{t} d t:=T_{0}(r, w)=T(r, w)+O(1)
$$

where

$$
S(r, w)=\frac{1}{\pi} \iint_{|\tilde{z}| \leq r}\left(\frac{\left|w^{\prime}(z)\right|}{1+|w(z)|^{2}}\right)^{2} d w .
$$

In this paper we use the Ahlfors-Shimizu characteristic in the same as the $T \underset{\sim}{\sim}, w)$ without any conflict with the following discussion. In general, suppose that $\widetilde{E}$ is a subset of $\widetilde{R}_{z}$; we denote

$$
S(r, E, w)=\frac{1}{\pi} \iint_{\widetilde{E}}\left(\frac{\left|w^{\prime}(z)\right|}{1+|w(z)|^{2}}\right)^{2} d w
$$

and

$$
T(r, E, w)=\frac{1}{v} \int_{0}^{r} \frac{S(t, E, w)}{t} d t
$$


The order and lower order of an algebroid function $w(z)$ are denoted by

$$
\lambda=\limsup _{r \rightarrow \infty} \frac{\log T(r, w)}{\log r}, \quad \mu=\liminf _{r \rightarrow \infty} \frac{\log T(r, w)}{\log r} .
$$

We define the angular domain

$$
\triangle\left(\theta_{0}, \delta\right)=\left\{z\left|\arg z-\theta_{0}\right|<\delta\right\}, 0 \leq \theta_{0}<2 \pi, 0<\delta<\frac{\pi}{2} .
$$

The part of $\widetilde{R}_{z}$ which lies over $\triangle\left(\theta_{0}, \delta\right)$ is denoted by $\widetilde{\triangle}\left(\theta_{0}, \delta\right)$. Let $n\left(r, \theta_{0}, \delta, a\right)$ (or $\left.\bar{n}\left(r, \theta_{0}, \delta, a\right)\right)$ be the number of zeros of $w(z)-a$ in $\widetilde{\triangle}\left(\theta_{0}, \delta\right) \cap\{|\widetilde{z}| \leq r\}$, counting multiplicities (or ignoring multiplicities). Put

$$
N\left(r, \theta_{0}, \delta, a\right)=\frac{1}{v} \int_{0}^{r} \frac{n\left(t, \theta_{0}, \delta, a\right)-n\left(0, \theta_{0}, \delta, a\right)}{t} d t+n\left(0, \theta_{0}, \delta, a\right) \log r .
$$

Let $\bar{n}^{l)}\left(r, \theta_{0}, \delta, a\right)$ be the number distinct zeros with multiplicity no greater than $l$ of $w(z)-a$ in $\widetilde{\triangle}\left(\theta_{0}, \delta\right) \cap\{|\widetilde{z}| \leq r\}$. Similarly, we can define $\bar{N}\left(r, \theta_{0}, \delta, a\right)$ and $\bar{N}^{l)}\left(r, \theta_{0}, \underset{\sim}{\delta}, a\right)$. Denote $n_{\chi}(r, w),\left(n_{\chi}\left(r, \triangle\left(\theta_{0}, \delta\right), w\right)\right.$ by the number of the branch points of $\widetilde{R}_{z}$ on $|z|<r$ (on the region $\triangle\left(\theta_{0}, \delta\right)$ ), counting the order of branch points. Denote

$$
N_{\chi}(r, w)=\frac{1}{v} \int_{0}^{r} \frac{n_{\chi}(t, w)-n_{\chi}(0, w)}{t} d t+\frac{n_{\chi}(0, w)}{v} \log r .
$$

Similarly, we can define $N_{\chi}\left(r, \triangle\left(\theta_{0}, \delta\right), w\right)$. We present some properties of $T(r, w)$ and $N_{\chi}(r, w)$.

LEMMA 2.1 [2].

$$
N_{\chi}(r, w) \leq 2(v-1) T(r, w)+O(1) .
$$

The following lemmas are useful in the proof of Theorem 1.5.

LEMMA 2.2. Suppose that $w(z)$ is a v-valued algebroid function in an angular domain $\triangle_{0}=\left\{z:|\arg z-\theta|<\delta_{0}\right\}$. Let $\bar{\triangle}=\{z:|\arg z-\theta| \leq \delta\}$ be an angular domain, contained in $\triangle_{0}$, where $\theta \in[0,2 \pi)$ and $0<\delta<\delta_{0}$. The part of $\widetilde{R}_{z}$ which lies over $\bar{\triangle}$ is denoted by $\underset{\triangle}{\triangle}$. Then for arbitrarily constant $\lambda>1$, positive integer $\alpha$ and any $q(q>2)$ distinct points $a_{1}, a_{2}, \ldots, a_{q} \in \bar{C}$,

$$
\begin{aligned}
\left(q-2-\frac{2}{l}\right) S(r, \bar{\triangle}, w) \leq & 2 \sum_{j=1}^{q} \bar{n}^{l)}\left(\lambda^{2 \alpha} r, \triangle_{0}, a_{j}\right)+\frac{l+1}{l}\left(1+\frac{1}{\alpha}\right) n_{\chi}\left(\lambda^{2 \alpha} r, \triangle_{0}, w\right) \\
& +\left(q-2-\frac{2}{l}\right) S\left(\lambda^{2 \alpha}, \bar{\triangle}, w\right)+O\left(\log ^{+} r\right) .
\end{aligned}
$$

Using Lemma 3.1 and the similar method in [12], we can prove that Lemma 2.2 is valid. We omit the details. 
LEMMA 2.3 [4]. Let $B(r)$ be a positive and continuous function in $[0,+\infty)$ which satisfies $\lim \sup _{r \rightarrow \infty}((\log B(r)) /(\log r))=\infty$. Then there exist continuously differentiable functions $\rho(r)$ and $U(r)$ which satisfy the following conditions.

(1) $\rho(r) \downarrow 0$ and $\rho^{\prime}(r)$ are monotone increasing.

(2) $\lim _{r \rightarrow \infty} r \rho^{\prime}(r) \log r \log \log r=0$.

(3) For sufficient large $r$, we have

$$
B(r) \ll U(r)=r^{\exp (1 /(\rho(r)))},
$$

where ' $\ll$ ' denotes that $B(r) \leq U(r)$ and there is a sequence $\left\{r_{n}\right\} \rightarrow \infty$, such that $B\left(r_{n}\right)=U\left(r_{n}\right)$.

(4) $U(R)<(1+o(1)) U(r)$, where $R=r+\left((r \log r) /\left(\log U(r) \log ^{2} \log U(r)\right)\right)$.

\section{Proof of Theorem 1.5}

PROOF. Assume that $w(z)$ is a $v$-valued algebroid function defined by (1.1) and satisfying (1.2). Let $m(m \geq 4)$ be a positive integer such that

$$
\theta_{0}=0, \quad \theta_{1}=\frac{2 \pi}{m}, \ldots, \quad \theta_{m-1}=\frac{(m-1) 2 \pi}{m}, \quad \theta_{m}=\theta_{0},
$$

and

$$
\Delta\left(\theta_{i}\right)=\left\{z:\left|\arg z-\theta_{i}\right|<\frac{2 \pi}{m}\right\}, \quad i=0,1, \ldots, m-1 ; \Delta\left(\theta_{m}\right)=\Delta\left(\theta_{0}\right) .
$$

Then among these $m$ angular domains $\left\{\triangle\left(\theta_{i}\right)\right\}$, there is at least one angular domain $\triangle\left(\theta_{i}\right)$ such that the relative expression

$$
\limsup _{r \rightarrow \infty} \frac{\bar{N}^{l)}\left(r, \Delta\left(\theta_{i}\right), a\right)}{T(r, w)}>0
$$

holds for all $a \in \mathbb{C}_{\infty}$ with at most $p$ exceptions; here $p$ and $l$ satisfy (1.3).

In the following, we need to consider two different cases.

(I) $\lim \inf _{r \rightarrow \infty}((\log T(r, w)) /(\log r))=\mu<+\infty$. In this case, we shall prove that (3.1) is valid by using a similar method to that in [8]. If it is not valid, then for any angular domain $\Delta\left(\theta_{i}\right)(1 \leq i \leq m)$, we have $q=p+1$ distinct points $a_{i}^{j}(j=1,2, \ldots, q)$ in $\mathbb{C}_{\infty}$ such that

$$
\sum_{i=0}^{m-1} \sum_{j=1}^{q} \bar{N}^{l)}\left(r, \triangle\left(\theta_{i+1}\right), a_{i+1}^{j}\right)=o(T(r, w)) .
$$

Let $\alpha$ be an arbitrary positive integer. Put

$$
\theta_{i, k}=\frac{2 \pi i}{m}+\frac{2 \pi k}{\alpha m}, \quad 0 \leq i \leq m-1,0 \leq k \leq \alpha-1, \theta_{i, 0}=\theta_{i} .
$$


For sufficiently large $r$, let

$$
\triangle_{i, k}=\left\{z:|z|<r, \theta_{i, k} \leq \arg z<\theta_{i, k+1}\right\} .
$$

Then

$$
\{|z|<r\}=\sum_{k=0}^{\alpha-1} \sum_{i=0}^{m-1} \triangle_{i, k} .
$$

Hence, there must be one $k_{0}\left(0 \leq k_{0} \leq \alpha-1\right)$, such that

$$
\sum_{i=0}^{m-1} n\left(\triangle_{i, k_{0}}, \widetilde{R}_{z}\right) \leq \frac{1}{\alpha} n\left(r, \widetilde{R}_{z}\right) .
$$

Define the angular domains

$$
\begin{gathered}
\bar{\triangle}_{i}=\left\{z \mid \frac{\theta_{i, k_{0}}+\theta_{i, k_{0}+1}}{2} \leq \arg z \leq \frac{\theta_{i+1, k_{0}}+\theta_{i+1, k_{0}+1}}{2}\right\}, \\
\triangle_{i}^{0}=\left\{z \mid \theta_{i, k_{0}}<\arg z<\theta_{i+1, k_{0}+1}\right\} \subset \triangle\left(\theta_{i+1}\right) .
\end{gathered}
$$

Since $\triangle_{i}^{0}$ only covers $\triangle_{i, k_{0}}$ twice,

$$
\sum_{i=0}^{m-1} n_{\chi}\left(r, \triangle_{i}^{0}, w\right) \leq\left(1+\frac{1}{\alpha}\right) n_{\chi}(r, w) .
$$

Hence,

$$
\sum_{i=0}^{m-1} N_{\chi}\left(r, \triangle_{i}^{0}, w\right) \leq\left(1+\frac{1}{\alpha}\right) N_{\chi}(r, w) .
$$

Applying Lemma 2.2 to $\triangle_{i}^{0}, \bar{\triangle}_{i}$,

$$
\begin{aligned}
\left(q-2-\frac{2}{l}\right) T\left(r, \bar{\triangle}_{i}, w\right) \leq & 2 \sum_{j=1}^{q} \bar{N}^{l)}\left(\lambda^{2 \alpha} r, \triangle_{i}^{0}, a_{i+1}^{j}\right) \\
& +\frac{l+1}{l}\left(1+\frac{1}{\alpha}\right) N_{\chi}\left(\lambda^{2 \alpha} r, \triangle_{i}^{0}, w\right)+O\left(\log ^{2} r\right) .(*)
\end{aligned}
$$

By $T(r, w)=\sum_{i=0}^{m-1} T\left(r, \bar{\triangle}_{i}, w\right)$, sum to $i=0,1, \ldots, m-1$ in two sides of the above expression (*) and substituting (3.4), we obtain

$$
\begin{aligned}
\left(q-2-\frac{2}{l}\right) T(r, w) \leq & 2 \sum_{i=0}^{m-1} \sum_{j=1}^{q} \bar{N}^{l)}\left(\lambda^{2 \alpha} r, \triangle_{i}^{0}, a_{i+1}^{j}\right) \\
& +\left(1+\frac{1}{\alpha}\right)^{2} \frac{l+1}{l} N_{\chi}\left(\lambda^{2 \alpha} r, w\right)+O\left(\log ^{2} r\right) .
\end{aligned}
$$


Applying (3.2) to (3.5) and then combining Lemma 2.1 shows that

$$
\begin{aligned}
\left(q-2-\frac{2}{l}\right) T(r, w) & \leq\left(1+\frac{1}{\alpha}\right)^{2} \frac{l+1}{l} N_{\chi}\left(\lambda^{2 \alpha} r, w\right)+O\left(\log ^{2} r\right) \\
& \leq 2(v-1)\left(1+\frac{1}{\alpha}\right)^{2} \frac{l+1}{l} T\left(\lambda^{2 \alpha} r, w\right)+O\left(\log ^{2} r\right) .
\end{aligned}
$$

By the hypothesis and applying [8, Lemma 1] to $T(r, w)$,

$$
\lim _{n \rightarrow \infty} \frac{T\left(r_{n}, w\right)}{\log ^{2} r_{n}}=+\infty, \quad T\left(\lambda^{2 \alpha} R_{n}, w\right) \leq \lambda^{2 \alpha \mu} T\left(R_{n}, w\right),
$$

where $R_{n}^{1-o(1)} \leq r_{n} \leq R_{n}(n \rightarrow \infty)$. From this,

$$
\lim _{n \rightarrow \infty} \frac{T\left(R_{n}, w\right)}{\log ^{2} R_{n}}=+\infty .
$$

In (3.6), we let $r=R_{n}$ and obtain

$$
\left(q-2-\frac{2}{l}\right) T\left(R_{n}, w\right) \leq 2(v-1)\left(1+\frac{1}{\alpha}\right)^{2} \frac{l+1}{l} \lambda^{2 \alpha \mu} T\left(R_{n}, w\right)+O\left(\log ^{2} R_{n}\right) .
$$

Hence,

$$
\left(q-2-\frac{2}{l}\right) \leq 2(v-1)\left(1+\frac{1}{\alpha}\right)^{2} \frac{l+1}{l} \lambda^{2 \alpha \mu} .
$$

Letting $\alpha \rightarrow \infty, \lambda \rightarrow 1$, we get

$$
\left(q-2-\frac{2}{l}\right) \leq 2 \frac{l+1}{l}(v-1) .
$$

Hence,

$$
\begin{cases}q \leq 4 v & \text { if } l=1 \\ q \leq 3 v & \text { if } l=2 \\ q \leq 2 \frac{l+1}{l} v & \text { if } 3 \leq l \leq 2 v \\ q<2 v+1 & \text { if } l \geq 2 v+1\end{cases}
$$

This contradicts $q=p+1$ and (3.1) follows.

(II) $\liminf r \rightarrow \infty((\log T(r, w)) /(\log r))=+\infty$, that is to say $w(z)$ is an infinite order function. By Lemma 2.3, there exists $U(r)$ satisfying conditions (1)-(4) of Lemma 2.3. By using the same method as that in [9], we can assert that there is at least an angular domain $\Delta\left(\theta_{i}\right)$ such that the relative expression

$$
\limsup _{r \rightarrow \infty} \frac{\bar{N}^{l)}\left(r, \triangle\left(\theta_{i}\right), a\right)}{U(r)}>0
$$

holds for all $a \in \bar{C}$ with at most $p$ exceptions. We omit the details. 
Furthermore,

$$
\begin{aligned}
\limsup _{r \rightarrow \infty} \frac{\bar{N}^{l)}\left(r, \triangle\left(\theta_{i}\right), a\right)}{T(r, w)} & =\limsup _{r \rightarrow \infty} \frac{\bar{N}^{l)}\left(r, \triangle\left(\theta_{i}\right), a\right)}{U(r)} \frac{U(r)}{T(r, w)} \\
& \geq \limsup _{r \rightarrow \infty} \frac{\bar{N}^{l)}\left(r, \triangle\left(\theta_{i}\right), a\right)}{U(r)} \liminf _{r \rightarrow \infty} \frac{U(r)}{T(r, w)}>0 .
\end{aligned}
$$

Hence, (3.1) follows in this case.

By (3.1), we can see that for arbitrary positive integer $m$ there exists an angular domain

$$
\triangle\left(\theta_{m}\right)=\left\{z:\left|\arg z-\theta_{m}\right|<\frac{2 \pi}{m}\right\}
$$

such that for any $a$,

$$
\limsup _{r \rightarrow \infty} \frac{\bar{N}^{l)}\left(r, \triangle\left(\theta_{m}\right), a\right)}{T(r, w)}>0,
$$

except for $p$ exceptions at most. Choosing a subsequence of $\left\{\theta_{m}\right\}$, and continuing to denote it by $\left\{\theta_{m}\right\}$, we assume that $\theta_{m} \rightarrow \theta$. Put $L: \arg z=\theta$. Then $L$ is the $T$ direction of Theorem 1.5.

In fact, for any $\varepsilon(0<\varepsilon<\pi / 2)$, when $m$ is sufficiently large, we have $\triangle\left(\theta_{m}\right) \subset$ $\triangle(\theta, \varepsilon)$. By (3.7), we obtain

$$
\limsup _{r \rightarrow \infty} \frac{\bar{N}^{l)}(r, \theta, \varepsilon, a)}{T(r, w)} \geq \limsup _{r \rightarrow \infty} \frac{\bar{N}^{l)}\left(r, \triangle\left(\theta_{m}\right), a\right)}{T(r, w)}>0
$$

with at most $p$ exceptions for $a$. Hence, Theorem 1.5 holds in this case.

\section{References}

[1] H. Guo, J. H. Zheng and T. W. Ng, 'On a new singular direction of meromorphic functions', Bull. Austral. Math. Soc. 69 (2004), 277-287.

[2] Y. Z. He and X. Z. Xiao, Algebroid Function and Ordinary Differential Equation (Science Press, Beijing, 1988).

[3] Y. N. Lü and Y.-X. Gu, 'On the existence of Borel direction for algebroid function', Kexue Tongbao Math. 28 (1983), 264-266.

[4] D. C. Sun, 'On the existence of Nevanlinna direction', Chinese Ann. Math. Ser. A 7 (1986), 212221 (in Chinese).

[5] N. Toda, 'Sur les directions de Julia et de Borel des fonctions algebroides', Nagoya Math. J. 34 (1969), 1-23.

[6] G. Valiron, 'Sur la dérivée des fonctions algébroïdes', Bull. Soc. Math. France 59 (1931), 17-39.

[7] - 'Sur les direction de Borel des fonctions algebroïdes meromorphes d'ordre infini', C. R. Acad. Sci. Paris 206 (1938), 735-737.

[8] S. M. Wang and Z. S. Gao, 'On a new singular direction of algebroid functions', Bull. Austral. Math. Soc. 75 (2007), 459-468.

[9] Z. J. Wu, 'On T direction of algebroidal functions', J. Math. Kyoto Univ. 47 (2007), 767-779. 
[10] Z. J. Wu and D. C. Sun, 'On the existence of T direction of meromorphic function concerning multiple values', Kodai Math. J. 31 (2008), 133-149.

[11] L. Yang, Value Distribution Theory and its New Research (Science Press, Beijing, 1982) (in Chinese); (Springer, Berlin, 1993) (in English).

[12] S. H. Zhang and D. C. Sun, 'On the singular direction of algebroid function', Southeast Asian Bull. Math. 30 (2006), 1179-1189.

[13] J. H. Zheng, 'On transcendental meromorphic functions with radially distributed values', Sci. China Ser. A 47 (2004), 401-416.

[14] Z.-X. Xuan, 'On the existence of $T$-direction of algebroid functions: a problem of J. H. Zheng', J. Math. Anal. Appl. 34 (2008), 540-547.

ZHAOJUN WU, Department of Mathematics, Xianning University, Xianning, Hubei 437100, PR China

e-mail: wuzj52@hotmail.com

DAOCHUN SUN, School of Mathematic, South China Normal University, Guangzhou, 510631, PR China

e-mail: sundch@scnu.edu.au 